\title{
HPM Generation by Tri-Anode Coaxial Vircator
}

\author{
H. ShaO*, G.Z. Liu, Y.P. Zhang, Z.F. Yang, J. Sun and Y.C. Zhang \\ Northwest Institute of Nuclear Technology, Shaanxi, Xi'an, 710024 China
}

\begin{abstract}
A novel type of coaxial vircator with 3 anodes is proposed. The advantages on high power microwave generation is tested by particle-in-cell simulation and theoretical analysis model is set up as well. The tri-anode coaxial vircator behaves a very stable frequency in the pulse duration. In the tri-anode coaxial vircator, the injecting electron beam is well premodulated in the first anode cavity and forms an inerratic bunch-dispersion mechanism in the second anode cavity; the energy-loss electrons penetrate through the third anode and absorbed by the dumping cavity locates in the centre electrode, which helps keeping the bunch-dispersion cycle undisturbed. A $3.8 \mathrm{GHz}$ tri-anode coaxial vircator is theoretically designed and optimized by particle-in-cell simulation, which gives beam-wave conversion efficiency of over $20 \%$ with a stable predominant frequency, in accordance with the particle-in-cell simulation and analytic model.
\end{abstract}

PACS numbers: $75.47 . \mathrm{Gk}$

\section{Introduction}

During the past few years, many novel types of virtual cathode oscillator (vircator) were designed to increase the beam power to high power microwave (HPM) power conversion efficiency. Among them, coaxial vircator is a potential device with the possibility to achieve the final goals. With a quasi-cavity configuration, the coaxial vircator has been tested for the capacity of relative high efficiency and stable frequency output compared with other virtual cathode oscillators [1-3]. For further increasing of the efficiency of coaxial vircator, a novel type of coaxial vircator with 3 anodes is proposed. In the tri-anode coaxial vircator, the injected electrons beam is well premodulated during its passing through the first anode cavity and forms an inerratic bunch-dispersion mechanism in the second anode cavity; the energy-loss electrons penetrate through the third anode and are absorbed by the cavity on centre electrode, which helps keeping the bunch-dispersion cycle undisturbed.

\section{Configuration and design of tri-anode coaxial vircator}

In the tri-anode coaxial vircator (shown in Fig. 1), the injected electrons beam passes through 2 coaxial cavities before it arrives at the dumping cavity on the centre electrode. It is well premodulated in the first anode cavity and forms an inerratic bunch-dispersion mechanism in the second anode cavity. The bunched electron beam passes through coaxial gap between the third anode foil and centre electrode and interacts with the TEM wave in the coaxial cavity. After the interaction process,

* corresponding author; e-mail: shaohaonint@163.com the energy-loss electrons penetrate through the mesh on the centre electrode and are absorbed by dumping cavity wall. Since the energy loss electron beam has rather low current which is under the space-charge-limiting current of the drifting space, few of the electrons can get a reverse velocity before reaching the dumping cavity wall. Hence, the beam-wave interaction process is kept undisturbed by the scattered electrons, which helps keeping the bunch-dispersion cycle undisturbed.

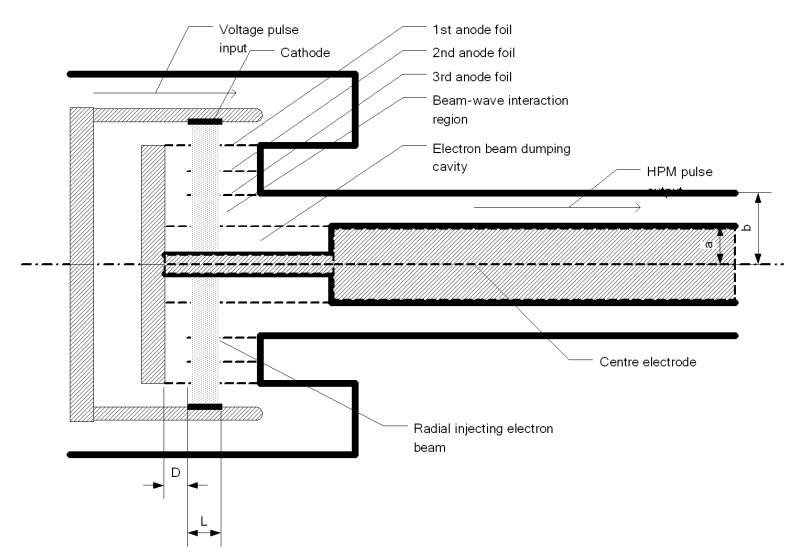

Fig. 1. Schematic view of tri-anode coaxial vircator.

For designing a tri-anode coaxial vircator the most important factor lies on the determination of the axial length and gaps between the anodes. The following is an example given on designing a $3.8 \mathrm{GHz}$ tri-anode coaxial vircator, by which we provide a practical model to describe the designing process. As is given in [5], for obtaining the maximal coupling efficiency between electron beam and the EM wave, the electron beam injecting region should be at the TEM wave node forms inside the 
coaxial cavity between the 1st and 2nd anodes. Accordingly, the wavelength of the operating modes inside anode cavity determined by shifting the exciting point from left to right along the axial direction of electron beam injecting region can be given as

$$
\frac{4 D}{2 n+1}<\lambda<\frac{4(D+L)}{2 n+1},
$$

which corresponds to a frequency band of $\Delta f=$ $\frac{c(2 n+1)}{4}\left(\frac{1}{D}-\frac{1}{D+L}\right)$, where $c$ is the speed of light, $D$ and $L$ are defined in Fig. 1, $n$ are the positive integer numbers $(1,2,3, \ldots)$. Within the frequency band $\Delta f$, interaction between injected electron beam and cavity mode can occur. The center frequency corresponding to the axial midpoint of injected electron region is

$$
f_{0}=\frac{c(2 n+1)}{4(D+L / 2)} \text {. }
$$

In order to decrease the effect of boundary distortion of electric field, $n$ should be selected equal or greater than 1 . If $n=1$, from Eq. (2) we have $D=3.15 \mathrm{~cm}$.

To obtain the same frequency of fast plasma wave of injected electron beam with operation frequency, the anode gap determined by the operation frequency can be combination of

$$
\left\{\begin{array}{l}
\bar{\tau}=\int_{r_{1}}^{r_{2}} \frac{\mathrm{d} r}{v}=\frac{2 \pi \varepsilon_{0}}{I_{n}} \boldsymbol{F}\left(r_{a}\right), \\
f_{0}=1 / 4 \bar{\tau},
\end{array}\right.
$$

where $\bar{\tau}, I_{n}$ are the transit time of injected electrons and electron beam current density on anode, respectively, and $F(r)=r \cdot E(r)$. The gap of 1 st and 2 nd anode foil calculated by Eqs. (3) is $1.5 \mathrm{~cm}$.

As described previously, the injected electron beam is premodulated by the $E$-field formed inside the gap between 1st and 2nd anode foil. For further modulating the electron beam, virtual cathode effect is introduced to increase the bunching of beam. The virtual cathode is formed between the 2nd and 3rd foil, where most electrons are decelerated periodically with its potential oscillation. Under the optimal condition, the transmitted electron beam can reach a $100 \%$ current modulation. Usually, to obtain a harmonic oscillation between the premodulation and virtual cathode modulation of electron beam, the gap between the 1st and 2nd foil is designed to be the same. The interaction cavity usually has a radial width of 2 to 2.5 times that of the anode gap.

The transmitted electron beam is deeply modulated by the anode gaps, and it couples with the $E$-field of TEM wave in the interaction cavity. For increasing the $E$-field in the interaction cavity, a feedback ring is introduced to reflect part of the output power back to the beam-wave interaction region. By this means, the power efficiency can be improved about $3-4 \%$.

From the electric field distribution character in the tri-anode coaxial vircator as shown in Fig. 2, we can also draw that, at the exciting frequency of $3.8 \mathrm{GHz}$, a preferable $E$-field forms inside the anode gaps and interaction region. At the injected beam region, radial $E$-field excited in the anode gaps premodulates the beam current,

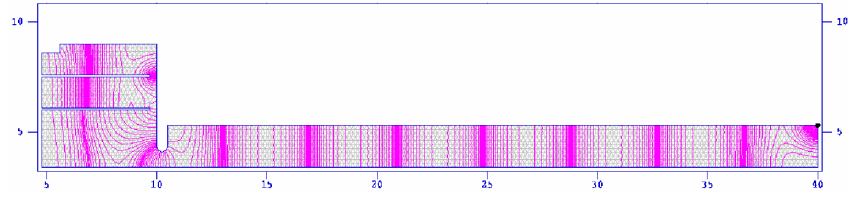

Fig. 2. Electric field distribution in the tri-anode coaxial vircator at the exciting frequency of $3.8 \mathrm{GHz}$.

then the bunched beam interacts with radial $E$-field in the coaxial cavity, and TEM wave gets growth for its preferable field distribution. Hence, TEM wave is expected to be the dominant mode in the output power.

\section{Particle-in-cell simulation}

After the parameter design according to the system analysis, particle-in-cell (PIC) simulation is carried out to test the design and optimize the parameters with code-CHIPIC [6]. Carefully changing the parameters of anode gaps, coupling slot and reflecting ring, we obtained a stable microwave output and narrow frequency span during the output pulse.

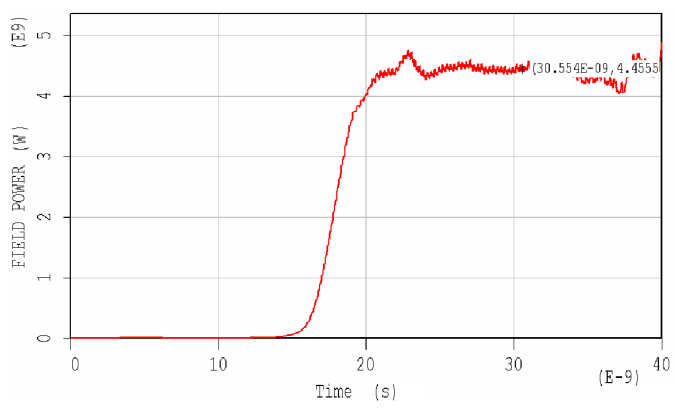

Fig. 3. Output power by PIC simulation.

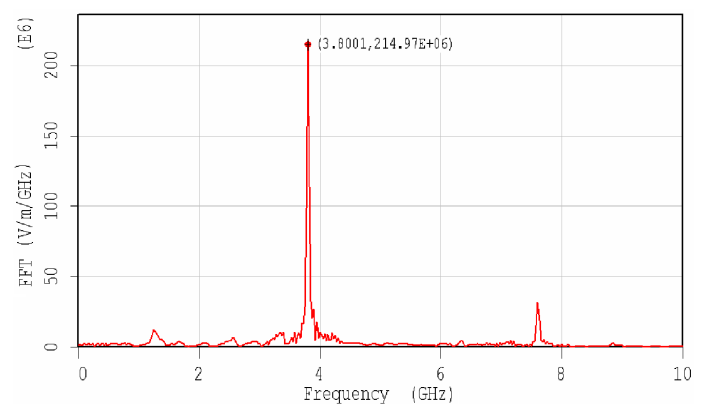

Fig. 4. High power microwave spectrum of tri-anode coaxial vircator.

An output power of $4.5 \mathrm{GW}$ (shown in Fig. 3) is obtained under $22 \mathrm{GW}$ input electron beam power, the power efficiency is over $20 \%$. Since the injected beam modulation forms after the coaxial anode cavities reach saturated excitation, the start time of tri-anode coaxial 
vircator is relatively longer than that of the usual vircators. It has 15 ns delay with the beam current rise time. The output power keeps stable for a plat input voltage pulse. The spectrum obtained in the PIC simulation as shown in Fig. 4 has a predominant frequency at $3.8 \mathrm{GHz}$, and the side band is evidently suppressed by effective decreasing of the energy loss electron beam reflection to keep the stable coupling process of beam-wave oscillation.

\section{Conclusion and discussion}

In the tri-anode coaxial vircator, the injected electron beam is well premodulated in the first coaxial anode cavity and forms an inerratic bunch-dispersion mechanism in the second anode cavity; the energy-loss electrons penetrate through the third anode and absorbed by centre electrode, which helps keeping the bunch-dispersion cycle undisturbed. The configuration of tri-anode coaxial vircator is proposed and the advantage on increasing the power efficiency is proved. Theoretical design process of a $3.8 \mathrm{GHz}$ system is discussed. PIC simulation with code-CHIPIC is carried out to test and optimize the configuration and input parameters. A power efficiency over $20 \%$ is obtained with a stable predominant frequency at $3.8 \mathrm{GHz}$.

\section{References}

[1] W. Jiang, W. Woolverton, J. Dickens, M. Kristiansen, in: Proc. IEEE International Conference on Plasma Science (ICOPS99), Monterey, CA, USA, p. 328 (1999).

[2] E.H. Choi, K.Y. Sung, W. Jeon, in: IEEE 30th International Conference on Vacuum Electronics, p. 341 (2003).

[3] H. Shao, G. Liu, Z. Yang, IEEE Trans. Plasma Sci. 34, 7 (2006).

[4] H. Shao, G. Liu, Z. Yang, J. Plasma Phys. 71, 563 (2005).

[5] H. Shao, G. Liu, Z. Yang, Z.M. Song, C.H. Chen, J. Sun, Y.P. Zhang, J. Plasma Phys. 74, 233 (2008).

[6] D. Liu, User's manual of code-CHIPIC, 2008.02. 